\title{
DEFORMATION OF DIRAC STRUCTURES ALONG ISOTROPIC SUBBUNDLES
}

\author{
IVÁN CALVO \\ Laboratorio Nacional de Fusión, Asociación EURATOM-CIEMAT, E-28040 Madrid, Spain \\ (e-mail: ivan.calvo@ciemat.es) \\ FERNANDO FALCETO \\ Departamento de Física Teórica and Instituto de Biocomputación y Física de Sistemas Complejos, \\ Universidad de Zaragoza, E-50009 Zaragoza, Spain \\ (e-mail: falceto@unizar.es) \\ and \\ MARCO ZAMBON \\ Universidade do Porto, Departamentos de Matematica Pura, \\ Rua do Campo Alegre 687, 4169-007 Porto, Portugal \\ (e-mail: mzambon@fc.up.pt) \\ (Received September 11, 2009 - Revised January 2, 2010)
}

\begin{abstract}
Given a Dirac subbundle and an isotropic subbundle of a Courant algebroid, we provide a canonical method to obtain a new Dirac subbundle. When the original Dirac subbundle is involutive (i.e. a Dirac structure) this construction has interesting applications, for instance to Dirac's theory of constraints and to the Marsden-Ratiu reduction in Poisson geometry.
\end{abstract}

Keywords: Courant algebroid, Dirac structure, Dirac's theory of constraints, reduction. 2000 MSC: 53D17, 53D99, 70H45.

\section{Introduction}

The concept of Dirac structure generalizes the Poisson and presymplectic structures by embedding them in the framework of the geometry of $T M \oplus T^{*} M$ or, more generally, the geometry of a Courant algebroid. Dirac structures were introduced in a remarkable paper by T. Courant [7]. Therein, they are related to the MarsdenWeinstein reduction [14] and to the Dirac bracket [9] on a submanifold of a Poisson manifold. More recently, Dirac structures have been considered in connection to the reduction of implicit Hamiltonian systems (see [1, 2]). This simple but powerful structure allows to deal with mechanical situations in which we have both gauge symmetries and Casimir functions.

We present a construction which takes an isotropic subbundle $S$ and a Dirac subbundle $D$ of an exact Courant algebroid, and produces a new Dirac subbundle $D^{S}$ 
(Definition 3.1). This construction, which we refer to as stretching, was introduced by the first two authors in [6]. When both $S$ and $D$ are involutive, we find conditions ensuring that $D^{S}$ is also involutive, i.e. a Dirac structure (Theorem 4.1).

We further show that three prominent classes of Dirac structures are indeed stretched Dirac structures: the Dirac brackets that appeared in Dirac's theory of constraints, the Dirac structures underlying the Marsden-Ratiu quotients in Poisson geometry [13], and coupling Dirac structures on Poisson fibrations [3].

The paper is organized as follows. In Section 2 we review basic definitions, in Section 3 we describe our stretching construction, in Section 4 we discuss when the stretched structure is involutive, and in Section 5 we present examples and applications.

\section{Courant algebroids and Dirac structures}

DEFINITION 2.1. A Courant algebroid [12] over a manifold $M$ is a vector bundle $E \rightarrow M$ equipped with an $\mathbb{R}$-bilinear bracket $[\cdot, \cdot]$ on $\Gamma(E)$, a nondegenerate symmetric bilinear form $\langle\cdot, \cdot\rangle$ on the fibers and a bundle map $\pi: E \rightarrow T M$ (the anchor) satisfying, for any $e_{1}, e_{2}, e_{3} \in \Gamma(E)$ and $f \in C^{\infty}(M)$ :

(i) $\left[e_{1},\left[e_{2}, e_{3}\right]\right]=\left[\left[e_{1}, e_{2}\right], e_{3}\right]+\left[e_{2},\left[e_{1}, e_{3}\right]\right]$,

(ii) $\pi\left(\left[e_{1}, e_{2}\right]\right)=\left[\pi\left(e_{1}\right), \pi\left(e_{2}\right)\right]$,

(iii) $\left[e_{1}, f e_{2}\right]=f\left[e_{1}, e_{2}\right]+\left(\pi\left(e_{1}\right) f\right) e_{2}$,

(iv) $\pi\left(e_{1}\right)\left\langle e_{2}, e_{3}\right\rangle=\left\langle\left[e_{1}, e_{2}\right], e_{3}\right\rangle+\left\langle e_{2},\left[e_{1}, e_{3}\right]\right\rangle$,

(v) $[e, e]=\mathcal{D}\langle e, e\rangle$,

where $\mathcal{D}: C^{\infty}(M) \rightarrow \Gamma(E)$ is defined by $\mathcal{D}=\frac{1}{2} \pi^{*} \circ \mathrm{d}$, using the bilinear form to identify $E$ and its dual.

We see from axiom $(\mathrm{v})$ that the bracket is not skew-symmetric but rather satisfies $\left[e_{1}, e_{2}\right]=-\left[e_{2}, e_{1}\right]+2 \mathcal{D}\left\langle e_{1}, e_{2}\right\rangle$.

A Courant algebroid is called exact if

$$
0 \longrightarrow T^{*} M \stackrel{\pi^{*}}{\longrightarrow} E \stackrel{\pi}{\longrightarrow} T M \longrightarrow 0
$$

is an exact sequence. Choosing a splitting $T M \rightarrow E$ of the above sequence with isotropic image allows one to identify the exact Courant algebroid with $T M \oplus T^{*} M$ endowed with the natural symmetric pairing

$$
\left\langle(X, \xi),\left(X^{\prime}, \xi^{\prime}\right)\right\rangle=\frac{1}{2}\left(i_{X^{\prime}} \xi+i_{X} \xi^{\prime}\right)
$$

and the Courant bracket

$$
\left[(X, \xi),\left(X^{\prime}, \xi^{\prime}\right)\right]=\left(\left[X, X^{\prime}\right], \mathcal{L}_{X} \xi^{\prime}-i_{X^{\prime}} \mathrm{d} \xi+i_{X^{\prime}} i_{X} H\right)
$$

for some closed 3-form $H$. In fact, the Courant algebroid uniquely determines the cohomology class of $H$, called Ševera class. The anchor $\pi$ is given by the projection onto the first component. When it is important to stress the value of the 3 -form $H$ 
we shall use the notation $E_{H}$ for $T M \oplus T^{*} M$ equipped with this Courant algebroid structure.

Definition 2.2. A Dirac subbundle or almost Dirac structure in an exact Courant algebroid $E$ is a subbundle $D \subset E$ which is maximal isotropic with respect to $\langle\cdot, \cdot\rangle$. The maximal isotropicity condition implies that $D^{\perp}=D$, where $D^{\perp}$ stands for the orthogonal subspace of $D$. In particular, $\operatorname{rank}(D)=\operatorname{dim}(M)$.

A Dirac structure is an involutive Dirac subbundle, i.e. a Dirac subbundle $D$ whose sections are closed under the Courant bracket. In this case the restriction to $D$ of the Courant bracket is skew-symmetric and $D$ with anchor $\pi$ is a Lie algebroid.

The two basic examples of Dirac structures are as follows.

EXAMPLE 2.1. For any 2-form $\omega$, the graph $L_{\omega}$ of $\omega^{b}: T M \rightarrow T^{*} M$ is a Dirac subbundle such that $\pi\left(L_{\omega}\right)=T M . L_{\omega}$ is a Dirac structure in $E_{H}$ if and only if $\mathrm{d} \omega=-H$. In particular, $L_{\omega}$ is a Dirac structure in $E_{0}$ if and only if $\omega$ is closed.

EXAMPLE 2.2. Let $\Pi$ be a bivector field on $M$. The graph $L_{\Pi}$ of the map $\Pi^{\sharp}: T^{*} M \rightarrow T M$ is always a Dirac subbundle. In this case the natural projection from $L_{\Pi}$ to $T^{*} M$ is one-to-one. $L_{\Pi}$ is a Dirac structure in $E_{H}$ if and only if $\Pi$ is a twisted Poisson structure. In particular, $L_{\Pi}$ is a Dirac structure in $E_{0}$ if and only it $\Pi$ is a Poisson structure.

\section{Stretched Dirac subbundles}

Until the end of this note we will assume the following setup:

$$
\begin{gathered}
E \text { is an exact Courant algebroid } \\
S \subset E \text { is an isotropic subbundle (i.e. } S \subset S^{\perp} \text { ) } \\
D \subset E \text { is a Dirac subbundle. }
\end{gathered}
$$

We further assume that $D \cap S$ (or equivalently $D \cap S^{\perp}$ ) has constant rank along $M$.

DEFINITION 3.1. The stretching of $D$ along $S[6]$ is the Dirac subbundle

$$
D^{S}:=\left(D \cap S^{\perp}\right)+S .
$$

To justify the fact that $D^{S}$ is maximal isotropic we use

$$
\begin{aligned}
\left(D^{S}\right)^{\perp} & =\left(D^{\perp}+S\right) \cap S^{\perp} \\
& =\left(D \cap S^{\perp}\right)+S=D^{S},
\end{aligned}
$$

where in the last line we have used that $D$ is maximal isotropic and $S$ is a subset of $S^{\perp}$. It is also clear that $D^{S}$, as the sum of two subbundles whose intersection has constant rank, is a (smooth) subbundle.

$D^{S}$ is the Dirac subbundle closest to $D$ among those containing $S$, as stated in the following proposition. 
Proposition 3.1. [6] Let $D, S$ and $D^{S}$ be as above and let $D^{\prime}$ be a Dirac subbundle such that $S \subset D^{\prime}$. Then, $D^{\prime} \cap D \subset D^{S} \cap D$. In addition, $D^{\prime} \cap D=D^{S} \cap D$ if and only if $D^{\prime}=D^{S}$.

Proof: From the isotropy of $D^{\prime}$ and $S \subset D^{\prime}$ we deduce that $D^{\prime} \subset S^{\perp}$. Hence,

$$
D^{\prime} \cap D \subset S^{\perp} \cap D=D^{S} \cap D .
$$

If the equality $D^{\prime} \cap D=D^{S} \cap D$ holds, then $D^{\prime} \supset D^{\prime} \cap D=S^{\perp} \cap D$. Since $S \subset D^{\prime}$, we find that $D^{S}=\left(D \cap S^{\perp}\right)+S \subset D^{\prime}$. But $D^{S}$ and $D^{\prime}$ have the same dimension, so that they are equal.

\section{Integrability}

In this section we determine various properties of $D^{S}$, in particular conditions under which $D^{S}$ is a Dirac structure.

Lemma 4.1. Assume that $S$ and $D$ are closed under the Courant bracket. Then the set of $S$-invariant sections of $D^{S}$,

$$
\left\{e \in D^{S}:[\Gamma(S), e] \subset \Gamma(S)\right\}
$$

is closed under the Courant bracket.

Proof: Consider two sections $e_{1}, e_{2} \in \Gamma\left(D^{S}\right)$ which are $S$-invariant, i.e. $\left[\Gamma(S), e_{i}\right]$ $\in \Gamma(S)$. First, let us prove that $\left[e_{1}, e_{2}\right]$ is an $S$-invariant section. Take $s \in \Gamma(S)$ and write

$$
\left[s,\left[e_{1}, e_{2}\right]\right]=\left[\left[s, e_{1}\right], e_{2}\right]+\left[e_{1},\left[s, e_{2}\right]\right]
$$

by Definition 2.1(i). Now recall that $[e, s]=-[s, e]$ for $e \in \Gamma\left(D^{S}\right)$ and $s \in \Gamma(S)$ because $D^{S}=(D+S) \cap S^{\perp} \subset S^{\perp}$. The $S$-invariance of $\left[e_{1}, e_{2}\right]$ follows immediately.

Next we show that $\left[e_{1}, e_{2}\right] \in \Gamma\left(D^{S}\right)$. Since we assumed that both $D \cap S^{\perp}$ and $S$ are subbundles, every section $e \in \Gamma\left(D^{S}\right)$ can be written as $e=v+w$ with $v \in \Gamma\left(D \cap S^{\perp}\right)$ and $w \in \Gamma(S)$. Notice that if $e$ is $S$-invariant, $v$ is also $S$-invariant because $S$ is Courant involutive. The expression

$$
\left[e_{1}, e_{2}\right]=\left[v_{1}+w_{1}, v_{2}+w_{2}\right]=\left[v_{1}, v_{2}\right]+\left[v_{1}, w_{2}\right]+\left[w_{1}, v_{2}\right]+\left[w_{1}, w_{2}\right]
$$

makes clear that $\left[e_{1}, e_{2}\right] \in \Gamma(D+S)$, since $\left[v_{1}, v_{2}\right] \in \Gamma(D)$ and the remaining terms on the right-hand side of Eq. (4.1) are sections of $S$. To prove that $\left[e_{1}, e_{2}\right] \in \Gamma\left(S^{\perp}\right)$ notice that, for any $s \in \Gamma(S)$,

$$
\left\langle s,\left[e_{1}, e_{2}\right]\right\rangle=\pi\left(e_{1}\right)\left\langle s, e_{2}\right\rangle-\left\langle\left[e_{1}, s\right], e_{2}\right\rangle=0,
$$

where we have used Definition 2.1(iv) as well as the orthogonality of $s$ and $e_{i}, \quad i=1,2$.

Inspired by [15] we will give the following definition. 
DEFINITION 4.1. Given a Dirac subbundle $D$ and an involutive isotropic subbundle $S \subset E$, we say that $S$ is canonical for $D$ if there exists a local $S$-invariant section ${ }^{1}$ of $D^{S}$ passing through any of its points.

Proposition 4.1. Assume that $S$ and $D$ are closed under the Courant bracket. We have the following chain of implications:

(a) $S$ is canonical for $D \Rightarrow$

(b) $D^{S}$ is a Dirac structure $\Rightarrow$

(c) $\left[\Gamma(S), \Gamma\left(D^{S}\right)\right] \subset \Gamma\left(D^{S}\right)$ (i.e. $S$ preserves $\left.D^{S}\right)$.

Proof: (a) $\Rightarrow$ (b): We have to show that the Courant bracket of two sections $v, v^{\prime} \in \Gamma\left(D^{S}\right)$ is again a section of $D^{S}$. We write $v$ and $v^{\prime}$ in terms of a local basis $\left\{e_{i}\right\}$ of $S$-invariant sections (such a basis always exists due to the canonicity of $S$ ). From Definition 2.1(iii) and Lemma 4.1 one immediately obtains that $\left[v, v^{\prime}\right]$ is a linear combination of the $e_{i}$ 's and hence belongs to $\Gamma\left(D^{S}\right)$.

(b) $\Rightarrow$ (c): holds because $S \subset D^{S}$.

The following theorem gives sufficient conditions to ensure that $D^{S}$ is a Dirac structure.

THEOREM 4.1. Assume that $S$ and $D$ are closed under the Courant bracket and additionally that $\pi\left(S^{\perp}\right)$ is an integrable regular distribution. Then items (a), (b) and (c) of Proposition 4.1 are all equivalent.

Proof: We just need to show that (c) $\Rightarrow$ (a) in Proposion 4.1. Notice first that $\pi(S)$ is a regular integrable distribution. Indeed,

$$
\operatorname{Ker}(\pi) \cap S=\pi^{*}\left(\pi\left(S^{\perp}\right)^{\circ}\right),
$$

and given that $\pi\left(S^{\perp}\right)$ is regular and $\pi^{*}$ is injective for exact Courant algebroids, it follows that $\operatorname{Ker}(\pi) \cap S$ is a subbundle. Now, the fact that $S$ is also a subbundle implies the regularity of $\pi(S)$. Integrability follows from the assumption that $\Gamma(S)$ is closed under the Courant bracket.

Take a commuting basis of local sections of $\pi(S)$ denoted by $\left\{\partial_{i}\right\}$. Fix lifts $s_{i}$ of $\partial_{i}$ to $S$, i.e. $s_{i} \in \Gamma(S)$ and $\pi\left(s_{i}\right)=\partial_{i}$. Since we are assuming (c) of Proposition 4.1 we can define a partial $\pi(S)$-connection on $D^{S}$ by imposing

$$
\nabla_{i} e:=\left[s_{i}, e\right] .
$$

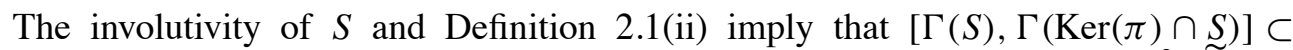
$\Gamma(\operatorname{Ker}(\pi) \cap S)$, so we can use $\nabla$ to define a partial $\pi(S)$-connection ${ }^{2} \widetilde{\nabla}$ on $D^{S} /(\operatorname{Ker}(\pi) \cap S)$. We now argue that $\widetilde{\nabla}$ is flat.

The curvature of the connection $\nabla$, with components $F_{i j}$, is given by

$$
F_{i j} e=\nabla_{i} \nabla_{j} e-\nabla_{j} \nabla_{i} e=\left[s_{i}\left[s_{j}, e\right]\right]-\left[s_{j}\left[s_{i}, e\right]\right]=\left[\left[s_{i}, s_{j}\right], e\right],
$$

and given that $\partial_{i}$ and $\partial_{j}$ commute and $S$ is involutive we have $\left[s_{i}, s_{j}\right] \in \operatorname{Ker}(\pi) \cap S$.

\footnotetext{
${ }^{1}$ Recall that a section $e$ is $S$-invariant iff $[\Gamma(S), e] \subset \Gamma(S)$.

${ }^{2}$ The connection $\widetilde{\nabla}$ depends on the choice of lifts $s_{i} \in \Gamma(S)$.
} 
Next we want to show that

$$
\left[\Gamma(\operatorname{Ker}(\pi) \cap S), \Gamma\left(D^{S}\right)\right] \subset \Gamma(\operatorname{Ker}(\pi) \cap S) .
$$

For that, take a section $s \in \Gamma(\operatorname{Ker}(\pi) \cap S)$ and write $s=\pi^{*}(\eta)$ with $\eta \in \Gamma\left(\pi\left(S^{\perp}\right)^{\circ}\right)$. Also take arbitrary sections $e \in \Gamma\left(D^{S}\right)$ and $s^{\perp} \in \Gamma\left(S^{\perp}\right)$. Now

$$
\left\langle[s, e], s^{\perp}\right\rangle=\left\langle\pi^{*}(\eta),\left[e, s^{\perp}\right]\right\rangle-\pi(e)\left\langle s, s^{\perp}\right\rangle=i_{\pi\left(\left[e, s^{\perp}\right]\right)} \eta=i_{\left[\pi(e), \pi\left(s^{\perp}\right)\right]} \eta=0,
$$

where in the first equality we used Definition 2.1 iv) and in last equality we used that $D^{S} \subset S^{\perp}$ and $\pi\left(S^{\perp}\right)$ is integrable.

Eq. (4.2) and Eq. (4.3) together imply that $\widetilde{\nabla}$ is a flat connection. Hence, through any point of $D^{S} /(\operatorname{Ker}(\pi) \cap S)$ passes a local horizontal sections for $\widetilde{\nabla}$, and any lift of it to a section $e_{h}$ of $D^{S}$ satisfies $\nabla_{i} e_{h} \in \Gamma(\operatorname{Ker}(\pi) \cap S)$ for all $i$. But the sections $\left\{s_{i}\right\}$ used to build the connection $\nabla$, together with $\Gamma(\operatorname{Ker}(\pi) \cap S)$, $\operatorname{span} \Gamma(S)$. Hence, using Definition 2.1(iii) and Eq. (4.3) we get

$$
\left[\Gamma(S), e_{h}\right] \subset \Gamma(S),
$$

completing the proof.

REMARK 4.1. With the hypotheses of Theorem 4.1, if $[\Gamma(S), \Gamma(D)] \subset \Gamma(D)$ then $S$ is canonical for $D$ (because condition (c) in Proposition 4.1 is satisfied). The converse is not true, see e.g. [15] for a counterexample in the context of Poisson manifolds.

\section{Examples and applications}

In this section we work only with the exact Courant algebroid $E_{0}$. The first two examples show that two well-known constructions in Poisson geometry, once phrased in tensorial terms, correspond to the stretching of Poisson structures.

\subsection{Dirac brackets}

We give a description of the classical Dirac bracket in tensorial terms, i.e. in terms of Dirac structures, thereby giving a clear geometric interpretation to the Dirac bracket. Further we present a natural generalization.

We recall first the construction of the Dirac bracket on a Poisson manifold $(M, \Pi)$.

DEFINITION 5.1. Given a regular foliation $R$ on an open set $U \subset M$ whose leaves $N$ are the level sets of second class constraints $\varphi^{1}, \ldots, \varphi^{m}$ (i.e. independent functions for which the matrix $C^{a b}:=\left\{\varphi^{a}, \varphi^{b}\right\}_{\Pi}$ is invertible, with the inverse $C_{a b}$ ), the Dirac bracket is defined as

$$
\{f, g\}_{\text {Dirac }}:=\{f, g\}_{\Pi}-\left\{f, \varphi^{a}\right\}_{\Pi} C_{a b}\left\{\varphi^{b}, g\right\}_{\Pi} .
$$

We denote by $\Pi_{\text {Dirac }}$ the bivector field corresponding to the bracket $\{\cdot, \cdot\}_{\text {Dirac }}$.

Lemma 5.1. (i) The level sets $N$ of $\varphi$ are cosymplectic submanifolds of $(M, \Pi)$ and therefore have a Poisson structure induced by $\Pi$. 
(ii) $\left(M, \Pi_{\text {Dirac }}\right)$ is obtained putting together the level sets $N$ of $\varphi$, endowed with the Poisson structure induced by П. In particular, the Dirac bracket (5.1) depends only on the level sets of the constraints (and not on the constraints themselves).

REMARK 5.1. (1) Here and in the following we use repeatedly the following fact: a Poisson (Dirac) manifold is determined by its foliation into symplectic (presymplectic) leaves.

(2) Lemma 5.1(ii) recovers the fact that (5.1) is a Poisson bracket.

Proof: (i) Since the $\varphi^{i}$ are second class constraints, the leaves $N$ of $R$ satisfy $\Pi^{\sharp} T N^{\circ} \oplus T N=\left.T M\right|_{N}$, which by definition means that they are cosymplectic submanifolds. There is an induced Poisson structure on $N$ [8, Section 8], obtained pulling back to $N$ the Dirac structure given by the graph of $\Pi$. The corresponding Poisson bracket of functions $f, g$ on $N$ is $\left.\{\tilde{f}, \tilde{g}\}_{\Pi}\right|_{N}$, where $\tilde{f}, \tilde{g}$ are extensions of $f, g$ to $M$ and $d \tilde{f}$ is required to annihilate $\Pi^{\sharp}\left(T N^{\circ}\right)$ at points of $N$.

(ii) One checks easily that $\left\{\varphi^{i}, g\right\}_{\text {Dirac }}=0$ for all $g \in C^{\infty}(U)$, i.e. that the $\varphi^{i}$ are Casimir functions for $\Pi_{\text {Dirac }}$, hence the level sets $N$ of $\varphi$ are Poisson submanifolds (i.e. unions of symplectic leaves) w.r.t. $\Pi_{\text {Dirac }}$. The Poisson structure on $N$ as a Poisson submanifold of $\left(M, \Pi_{\text {Dirac }}\right)$ agrees with the one induced by $\Pi$ in the way described in (i). Indeed, for all functions $f, g$ on $N$ and extensions $\tilde{f}, \tilde{g}$ as above we have $\left.\{\tilde{f}, \tilde{g}\}_{\text {Dirac }}\right|_{N}=\left.\{\tilde{f}, \tilde{g}\}_{\Pi}\right|_{N}$ since $\left.\left\{\tilde{f}, \varphi^{a}\right\}_{\Pi}\right|_{N}=0$ for all constraints $\varphi^{a}$.

Now consider an integrable distribution $R \subset T M$ and let $D$ be a Dirac structure on $E_{0} \rightarrow M$ so that $D \cap R^{\circ}$ has constant rank $^{3}$. We consider the stretched Dirac subbundle $D^{R^{\circ}}$.

The following proposition shows that in the special case that $D$ is the graph of a Poisson structure $\Pi$ and the leaves of $R$ are cosymplectic in $(M, \Pi)$, the Dirac subbundle $D^{R^{\circ}}$ gives exactly the classical Dirac bracket (Definition 5.1). Hence $D^{R^{\circ}}$ can be considered as a generalization of the classical Dirac bracket.

Proposition 5.1. (1) $D^{R^{\circ}}$ is a Dirac structure. It is constructed putting together the integral submanifolds $N$ of $R$, with the (smooth) Dirac structure induced pulling back $D$.

Now assume that $D$ is the graph of a Poisson structure $\Pi$.

(2a) $D^{R^{\circ}}$ is itself the graph of a Poisson structure iff the leaves of the distribution $R$ are Poisson-Dirac submanifolds [8] of $(M, \Pi)$.

(2b) Suppose the stronger condition that the leaves of $R$ are cosymplectic submanifolds of $(M, \Pi)$, so that the Dirac bracket (5.1) can be defined (see Lemma 5.1). Then $D^{R^{\circ}}$ is the graph of the Poisson structure $\Pi_{\text {Dirac }}$.

Proof: (1) Notice that since $R$ is integrable we can choose a frame for $R^{\circ}$ consisting of closed 1-forms, which act trivially under the Courant bracket. Hence

\footnotetext{
${ }^{3}$ Here $R^{\circ} \subset T^{*} M$ denotes the annihilator of $R$, i.e. the sections of $R^{\circ}$ are the 1 -forms that kill all sections of $R$.
} 
$R^{\circ}$ is canonical for $D$ (see Definition 4.1). So from Proposition 4.1 we conclude that $D^{R^{\circ}}$ is a Dirac structure.

Since $\pi\left(D^{R^{\circ}}\right)$ is everywhere tangent to the foliation $R$, the integral submanifolds $N$ of $R$ are unions of presymplectic leaves of $D^{R^{\circ}}$. The Dirac structure $D$ can be restricted to any leaf $N$ of the foliation induced by $R$, delivering a smooth subbundle since $D \cap R^{\circ}$ has constant rank [7] [5]. Further, a simple computation shows that the pullback to $N$ of $D$ is equal to the pullback to $N$ of $D^{R^{\circ}}$.

(2a) The Dirac structure $D^{R^{\circ}}$ is the graph of a bivector field if and only if $D^{R^{\circ}}+T M=T M \oplus T^{*} M$. Taking orthogonals we obtain $\left(D+R^{\circ}\right) \cap R=\{0\}$. This can be rewritten as $\Pi^{\sharp}\left(R^{\circ}\right) \cap R=\{0\}$, which by definition [8, Section 8] means that the leaves of $R$ are Poisson-Dirac submanifolds of $(M, \Pi)$.

(2b) This follows from Lemma 5.1(ii) and part (i) of this proposition.

REMARK 5.2. Proposition 5.1(2a) shows that even within the framework of Poisson geometry, i.e. in the case that both $D$ and $D^{R^{\circ}}$ correspond to Poisson structures, our construction of Dirac structure $D^{R^{\circ}}$ is more general than the classical Dirac bracket.

\subsection{The Marsden-Ratiu reduction}

We show that the reduced Poisson structure induced via Marsden-Ratiu reduction [13] from a Poisson manifold $(M, \Pi)$ is obtained pushing forward not $\Pi$ itself but rather a suitable stretching of $\Pi$.

We start by recalling the Poisson reduction by distributions as it was stated by Marsden and Ratiu in [13], see also [15]. The set-up is the following:

$(M,\{\cdot, \cdot\})$ is a Poisson manifold,

$N$ is a submanifold with embedding $\iota: N \hookrightarrow M$,

$B \subset T_{N} M$ is a smooth subbundle of $T M$ restricted to $N$.

We shall also assume that $F:=B \cap T N$ is an integrable regular distribution on $N$ and $\underline{N}:=N / F$ is a smooth manifold.

Definition 5.2. [13] $(M,\{\cdot, \cdot\}, N, B)$ is Poisson reducible if there is a Poisson bracket $\{\cdot, \cdot\}_{\underline{N}}$ on $\underline{N}$ such that for any $f_{1}, f_{2} \in C^{\infty}(\underline{N}) \cong C^{\infty}(N)_{F}$ we have

$$
\left\{f_{1}, f_{2}\right\}_{\underline{N}}=\iota^{*}\left\{f_{1}^{B}, f_{2}^{B}\right\}
$$

for all extensions $f_{i}^{B} \in C^{\infty}(M)_{B}$ of $f_{i}$.

Here $C^{\infty}(N)_{F}:=\left\{f \in C^{\infty}(N)|\mathrm{d} f|_{F}=0\right\}$ and $C^{\infty}(M)_{B}:=\left\{f \in C^{\infty}(M) \mid\right.$ $\left.\left.\mathrm{d} f\right|_{B}=0\right\}$.

Given $(M,\{\cdot, \cdot\}, N, B)$ clearly there is at most ${ }^{4}$ one Poisson bracket $\{\cdot, \cdot\}_{N}$ on $\underline{N}$ satisfying the requirement of Definition 5.2. The following proposition (which is

\footnotetext{
${ }^{4}$ References [13], and subsequently [10], formulate conditions which ensure that $(M,\{\cdot, \cdot\}, N, B)$ is Poisson reducible.
} 
essentially [10, Proposition A.2]) describes the reduced Poisson structure on $\underline{N}$ in terms of bivector fields rather than in terms of brackets: it is obtained from $\bar{\Pi}$ by stretching along $B$, pulling back to $N$ and then pushing forward to $\underline{N}$.

PROPOSITION 5.2. Assume that the prescription of Definition 5.2 gives a welldefined bivector field on $\underline{N}$, denote by $L_{N}$ its graph, and denote $L_{\Pi}=\operatorname{graph}(\Pi)$. Then the pullback of the almost Dirac structure $L_{\underline{N}}$ under $p: N \rightarrow \underline{N}$ is $\iota^{*}\left(L_{\Pi}^{B}\right)$.

Consequently, $L_{N}$ is given by the push-forward under $p$ of $\iota^{*}\left(L_{\Pi}^{B}\right)$.

\subsection{Couplings on Poisson fibrations}

Given a Dirac subbundle $D$ and an isotropic subbundle $S$, there are situations in which one wants to "deform" $D$ to a new Dirac subbundle which contains $S$. A natural candidate for the new Dirac structure is the stretching $D^{S}$. An instance is provided by our next example, inspired by the results of Brahic and Fernandes [3] which (in the case of a flat connection) can be rephrased in our formalism.

Our data are a manifold $M$ and:

A splitting of the tangent bundle into two regular, integrable distributions, $T M=$ Hor $\oplus$ Vert.

A two form in Hor: $\omega \in \Omega^{2}$ (Hor).

A bivector field in Vert: $\pi_{V} \in \wedge^{2}$ (Vert).

The question is how to combine these data and which are the conditions that produce a Dirac structure. In principle there are two dual ways of doing this by using the deformation by stretching. The two different procedures give the same result.

(a) Consider $\pi_{V}^{\sharp}: T^{*} M \longrightarrow$ Vert and take $D=\operatorname{graph}\left(\pi_{\mathrm{V}}^{\sharp}\right)$.

$D$ is a Dirac structure if and only if

(i) $\left[\pi_{V}, \pi_{V}\right]=0$.

To define $S$, the stretching direction, consider the bundle map $\hat{\omega}^{b}:$ Hor $\longrightarrow$ Vert $^{\circ}$ induced by $\omega$ and take $S=\operatorname{graph}\left(\hat{\omega}^{b}\right)$.

$S$ is involutive if and only if:

(ii) $\omega$ is horizontally closed,

(iii) $\mathcal{L}_{v}\left(\omega\left(u_{1}, u_{2}\right)\right)=0$ for $v \in \Gamma$ (Vert) and $u_{i} \in \Gamma$ (Hor) s. t. $\left[v, u_{i}\right] \in \Gamma$ (Vert).

Now, assuming that the other conditions hold, we can show that $S$ preserves $D^{S}$ if and only if

(iv) $\mathcal{L}_{u} \pi_{V}=0$ for any $u \in \Gamma$ (Hor) s. t. $[v, u] \in \Gamma$ (Vert), $\forall v \in \Gamma$ (Vert).

If conditions (i)-(iv) are satisfied then, using Theorem $4.1\left(S^{\perp}=S+\right.$ Vert + Hor $^{\circ}$, and therefore $\left.\pi\left(S^{\perp}\right)=T M\right)$, we have that $D^{S}$ defines a Dirac structure. In the next paragraph we shall show an alternative way of obtaining the same result with the roles of $\omega$ and $\pi_{V}$ exchanged. 
(b) We introduce first the bundle map $\omega^{b}:$ Hor $\longrightarrow$ Hor*.

Consider the Dirac subbundle $D^{\prime} \subset T M \oplus T^{*} M$ induced by graph $\left(\omega^{b}\right)$, i.e.

$$
D^{\prime}=\left\{(v, \xi) \mid v \in \text { Hor, }\left.\xi\right|_{\text {Hor }}=\omega^{b} v\right\} .
$$
holds.

One can show that $D^{\prime}$ is a Dirac structure if and only if condition $i$ i) above

Now we proceed to define the new stretching subbundle $S^{\prime}$. Take

$$
S_{x}^{\prime}=\left\{\left(\left(\pi_{V} \xi\right)_{x}, \xi_{x}\right) \mid \xi_{x} \in\left(\text { Hor }^{\circ}\right)_{x}\right\} .
$$

$S^{\prime}$ is involutive if and only if conditions $i$ ) and $i v$ ) hold.

Finally one can show that, assuming all previous conditions, the stretching $D^{\prime S^{\prime}}$ is a Dirac structure if and only if

(iii) $^{\prime} \mathcal{L}_{v}\left(\omega\left(u_{1}, u_{2}\right)\right)=0$ for $v \in \Gamma\left(\pi_{V}^{\sharp}\left(T^{*} M\right)\right)$ and $u_{i} \in \Gamma$ (Hor) s. t. $\left[v, u_{i}\right] \in$ $\Gamma$ (Vert).

It is interesting to compare the two constructions. First it is clear that $D^{S}=D^{\prime S^{\prime}}$. Further, condition (iii) in construction a) implies condition (iii) ${ }^{\prime}$ in (b). Therefore, even if both give the same final result, the second construction has a broader range of application.

REMARK 5.3. We establish the connection between the above and the coupling of Poisson fibrations of [3]. Suppose that $M$ is the total space of a fibration so that Vert is the distribution tangent to the fibers. One computes easily that $D^{S}$ agrees with the fiber nondegenerate almost Dirac structure associated to the triple $\left(\pi_{V}\right.$, Hor, $\left.\omega\right)$ in Corollary 2.6 of [3]. Brahic and Fernandes compute the necessary and sufficient conditions for this to be a Dirac structure in Corollary 2.8 of [3]. If the horizontal connection is flat their conditions are equivalent to our (i), (ii), (iii) $^{\prime}$ and (iv) above, i.e. the conditions for having a Dirac structure following the stretching procedure introduced in the paper.

\section{Acknowledgments}

Research partially supported by grants FPA2003-02948 and FPA2006-02315, MEC (Spain) and by the Forschungskredit 2006 of the University of Zürich.

\section{REFERENCES}

[1] G. Blankenstein and T. S. Ratiu: Singular reduction of implicit Hamiltonian systems, Rep. Math. Phys. 53 (2004), 211-260.

[2] G. Blankenstein and J. van der Schaft: Symmetry and reduction in implicit generalized Hamiltonian systems, Rep. Math. Phys. 47 (2001), 57-100.

[3] O. Brahic and R.L. Fernandes: Poisson Fibrations and Fibered Symplectic Groupoids, Contemporary Mathematics 450 (2008), 41-60.

[4] H. Bursztyn, G. Cavalcanti and M. Gualtieri: Reduction of Courant algebroids and generalized complex structures, Adv. Math. 211, no. 2 (2007), 726-765.

[5] H. Bursztyn, A. Weinstein: Poisson geometry and Morita equivalence, London Math. Soc. Lecture Note Ser. 323 (2005), 1-78. 
[6] I. Calvo and F. Falceto: Reduction and projection of Dirac structures, Monografías de la Real Academia de Ciencias de Zaragoza 29 (2006), 49-56.

[7] T. Courant: Dirac manifolds, Trans. Amer. Math. Soc. 319 (1990), 631-661.

[8] M. Crainic and R. Fernandes: Integrability of Poisson brackets, J. Differential Geom. 66, no. 1 (2004), 71-137.

[9] P. A. M. Dirac: Lectures on quantum mechanics, Yeshiva University, New York 1967.

[10] F. Falceto and M. Zambon: An extension of the Marsden-Ratiu reduction for Poisson manifolds, Lett. Math. Phys. 85, no. 2-3 (2008), 203-219.

[11] Z. J. Liu: Some remarks on Dirac structures and Poisson reductions, Banach Center Publ. 51 (2000), 165-173.

[12] Z. J. Liu, A. Weinstein and P. Xu: Manin triples for Lie bialgebroids, J. Differential Geom. 45, no. 3 (1997), 547-574.

[13] J. E. Marsden and T. Ratiu: Reduction of Poisson manifolds, Lett. Math. Phys. 11 (1986), 161-169.

[14] J. E. Marsden and A. Weinstein: Reduction of symplectic manifolds with symmetry, Rep. Math. Phys. 5 (1974), 121-130.

[15] J. P. Ortega and T. Ratiu: Momentum Maps and Hamiltonian Reductions, Birkhäuser, Basel-Boston-Berlin 2004.

[16] I. Vaisman: Isotropic subbundles of $T M \oplus T^{*} M$, Int. J. Geom. Methods Mod. Phys. 4, no. 3 (2007), 487-516.

[17] I. Vaisman: Weak-Hamiltonian dynamical systems, J. Math. Phys. 48, no. 8 (2007).

[18] M. Zambon: Reduction of branes in generalized complex geometry, J. Symplectic Geom. 6, no. 4 (2008), $353-378$. 